\title{
Adenylyl cyclase-associated protein 1-targeted nanoparticles as a novel strategy for the treatment of metastatic non-small cell lung cancer
}

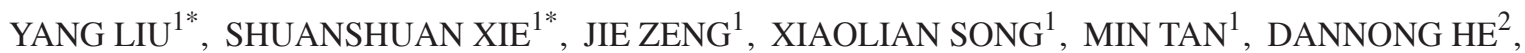 \\ JIE WANG $^{2}$, PING WANG ${ }^{2}$, JUN ZHU ${ }^{2}$ and CHANGHUI WANG ${ }^{1}$ \\ ${ }^{1}$ Department of Respiratory Medicine, Shanghai Tenth People's Hospital, Tongji University, Shanghai 200072; \\ ${ }^{2}$ National Engineering Research Center for Nanotechnology, Shanghai 200241, P.R. China
}

Received October 7, 2018; Accepted May 31, 2019

DOI: 10.3892/ijo.2019.4822

\begin{abstract}
Non-small cell lung cancer (NSCLC) is one of the most fatal cancers worldwide. Adenylyl cyclase-associated protein 1 (CAP1) belongs to a family of cyclase-associated proteins that are involved in the development of cancerous tumors. A previous study by our group confirmed the association between CAP1, lung cancer and the metastasis of cancer cells. In the present study, poly(lactic-polyglycolic acid; PLGA)/CAP1-small interfering (si)RNA nanoparticles were prepared and delivered into A549 cells. The performance of PLGA/siCAP1-siRNA nanoparticles for siRNA delivery was measured based on the results of migration assay and animal experiments. The multifunctional nanoparticles were determined to be capable of inhibiting CAP1 expression, which reduced NSCLC metastasis in vitro and in vivo. Therefore, the findings of the current study highlighted the potential use of PLGA/siCAP1-siRNA nanoparticles for the treatment of NSCLC metastasis.
\end{abstract}

\section{Introduction}

Lung cancer has been recognized as the most serious human disease worldwide (1). Based on the online database

Correspondence to: Professor Changhui Wang, Department of Respiratory Medicine, Shanghai Tenth People's Hospital, Tongji University, 301 Middle Yanchang Road, Shanghai 200072, P.R. China E-mail: wang-chang-hui@hotmail.com

*Contributed equally

Abbreviations: CAP1, adenylyl cyclase-associated protein 1; NSCLC, non-small cell lung cancer; NP, nanoparticles; siRNA, small interfering RNA; PLGA, poly(lactic-polyglycolic acid); PLGA/siCAP1, CAP1-siRNA in PLGA; PLGA/Ctrl, control-siRNA in PLGA; RT-qPCR, reverse transcription-quantitative polymerase chain reaction; FBS, fetal bovine serum; CCK-8, cell counting kit-8

Key words: non-small cell lung cancer, metastasis, poly(lacticpolyglycolic acid), nanoparticles, adenylyl cyclase-associated protein 1
GLOBOCAN, lung cancer was the most commonly diagnosed and severest cancer all over the world in 2013, accounting for $13 \%$ of all the diagnosed cancers and $19.4 \%$ of all cancer-associated mortalities in 184 nations and regions (2). Nearly $85 \%$ of all lung cancer cases are non-small cell lung cancer (NSCLC) cases, including bronchioloalveolar cancer, large cell cancer, adenocarcinoma and squamous cell cancer (2). Over $50 \%$ of patients with NSCLC developed metastasis following diagnosis, with only $14 \%$ surviving for at least 5 years $(3,4)$. Chemotherapy resistance, aggressive tumor growth and distant metastasis are the primary contributors to NSCLC-associated mortality worldwide $(3,4)$.

Adenylyl cyclase-associated protein 1 (CAP1) is an actin monomer-binding protein coded by a gene termed CAPl (5), which was initially cloned in budding yeast and is located downstream of RAS (6). The human homolog of CAP1 was first identified in the early 1990s (7). CAPs are associated with actin in various organisms ranging from yeast to mammals (8). In addition, CAP1 has been demonstrated to serve a crucial role in accelerating actin filament turnover (9). Based on previous findings demonstrating the crucial roles of actin filaments in regulating CAP1 expression and cell migration $(10,11)$, studies further confirmed the close association between CAP1 expression and tumor metastasis (12-14). Previous findings by our group indicated that CAP1 expression is upregulated in patients with NSCLC compared with that in healthy individuals (15-17). Furthermore, CAP1 expression was demonstrated to be upregulated in patients with metastatic NSCLC compared with that in patients with non-metastatic NSCLC $(15,16)$. Therefore, CAP1 expression could be used to predict metastasis in and the prognosis of patients with (18).

RNA inference (i)-mediated gene-silencing in mammals can effectively inhibit gene expression at transcriptional, post-transcriptional and translational levels (19). RNAi has been recently employed for the selective interference of specific gene expression via the introduction of artificially synthesized small interfering (si)RNAs (19). However, the entry of siRNAs into the target cells is barely feasible without a suitable carrier (20). Nanoparticles (NPs) have demonstrated good performances in the delivery of siRNAs to silence key genes and inhibit the progression of disease in animal models, 
thereby highlighting their potential application in human clinical trials (20-22). Additionally, poly(lactic-co-glycolic acid; PLGA) is an ideal non-toxic and non-immunostimulatory vehicle for delivering siRNAs (23-25).

\section{Materials and methods}

Materials. The following reagents were used in the present study: Poly(lactide-co-glycolide) Resomer RG502 [PLGA-COOH; molecular weight (MW), 20,000; Jinan Daigang Biomaterial Co., Ltd., Jinan, China], A549 and H1299 cells (Cell Bank of the Chinese Academy of Sciences), RPMI-1640 medium, fetal bovine serum (both Gibco; Thermo Fisher Scientific, Inc.), Cell Counting Kit (CCK)-8 (Dojindo Molecular Technologies, Inc.), amine-poly(ethylene glycol)-carboxymethyl ( $\mathrm{NH}_{2}-\mathrm{PEG}-\mathrm{COOH}$; MW, 3,400) was purchased from Seebio Biotech (Shanghai) Co., Ltd. Triethylamine and dichloromethane were purchased from Sinopharm Chemical Reagent Co., Ltd.. All chemical reagents were analytical grade or above. Nude mice were obtained from the Experimental Animal Center of Shanghai Tenth People's Hospital of Tongji University (Shanghai, China).

Synthesis of PLGA-PEG macromolecule. Triethylamine and dichloromethane were dried with calcium hydride before use as described previously (26). Carboxylate-functionalized copolymer PLGA-PEG was synthesized by the conjugation of PLGA-COOH and $\mathrm{NH}_{2}$-PEG-COOH. Briefly, $200 \mathrm{mg}$ PLGA-COOH, 2.9 mg 1-(3-Dimethylaminopropyl)-3-ethylcarbodiimide hydrochloride (Sigma-Aldrich; Merck KGaA), $1.8 \mathrm{mg}$ N-Hydroxysuccinimide (NHS; Sinopharm Chemical Reagent Co., Ltd.) and $1 \mathrm{mg}$ 4-dimethylaminopyridine (Sinopharm Chemical Reagent Co., Ltd.) were dissolved in $10 \mathrm{ml}$ dichloromethane, and stirred for $24 \mathrm{~h}$ at room temperature to convert PLGA-COOH to PLGA-NHS. Then, $34 \mathrm{mg}$ $\mathrm{NH}_{2}$-PEG-COOH and $8.6 \mu \mathrm{l}$ triethylamine were added into the solution. After further stirring for $8 \mathrm{~h}$ at room temperature, dichloromethane was removed through the use of a rotary evaporator and $5 \mathrm{ml} \mathrm{N}$,N-Dimethylformamide (Sinopharm Chemical Reagent Co., Ltd.) was added to dissolve PLGA-PEG. Then, the solution was added to a dialysis bag (width, 44 mm; MW cut-off, 8,000-14,000); unreacted PEG and other small molecules were removed by dialyzing for 3 days, and then PLGA-PEG was freeze-dried for 24-48 h.

Preparation of PLGA/siCAP1 NPs. PLGA/siCAP1 NPs were prepared by the revised solvent emulsion/evaporation method to obtain capsules with a polymeric shell that encapsulates siCAP1 (Shanghai GenePharma Co., Ltd., Shanghai, China) as previously described (27). The sequence for the siCAP1 was 5'-GGAGCCAGCUGUACUUGAATT-3' and reverse, 5'-UUCAAGUACAGCUGGCUCCTT-3'. The sequences of negative control siRNA were: 5'-UUCUCCGAACGUGUCACGUTT-3' (sense) and 5'-ACGUGACACGUUCGGAGA ATT-3' (antisense).

Briefly, $10 \mathrm{mg}$ PLGA-PEG was dissolved in a solvent mixture containing $200 \mu \mathrm{l}$ dichloromethane, $200 \mu 1$ 15\% (w/v) Span80 dichloromethane (Shenzhen Sheng Xin Automation Equipment Co., Ltd., Shenzhen, China) along with $16 \mu \mathrm{g}$ siCAP1. Following a thorough mixing, the resulting mixture was emulsified using $\mathrm{T} 10$ basic ULTRA-TURRAX ${ }^{\circledR}$ (IKA England Ltd.) to produce a pre-emulsion with a final volume of $2 \mathrm{ml}$. A 15-ml centrifuge tube was used to emulsify the solution on ice for $1 \mathrm{~min}$. Subsequently, an ultrasonic homogenizer/probe sonicator with a vibrating metallic tip (model no. JY 92-IIN; tip diameter, 3 mm; Ningbo Scientz Biotechnology Co., Ltd.) was used to sonicate the emulsion on ice for $3 \mathrm{sec}$, followed by a $10 \mathrm{sec}$ of pause; the process was repeated several times for 3 min each under $50 \%$ power $(300 \mathrm{~W})$. After an additional $10 \mathrm{~min}$ of stirring (cycle, 1 min of stirring and a 1-min pause) using T 10 basic ULTRA-TURRAX on ice, the organic solvents were subjected to $3 \mathrm{~h}$ of evaporation with magnetic stirring at room temperature (27-30). To contrast the PLGA-PEG/siCAP1 NPs (PLGA/siCAP1), a PLGA/negative control siRNA (Ctrl; Shanghai GenePharma Co., Ltd.), a PLGA emulsion alone and PLGA-fluorescein isothiocyanate (FITC; Shanghai GenePharma Co., Ltd.) were prepared using the same process. In addition, PLGA mixed with PLGA-FITC $[1: 1(\mathrm{w} / \mathrm{w})]$ was used to prepare the emulsion for in vivo bio-distribution imaging.

siCAP1 binding and gel retardation assay. Increasing amounts of PLGA (w/w) were complexed to a fixed amount of siCAP1 (500 ng) in OptiMEM I reduced serum culture medium (Gibco; Thermo Fisher Scientific, Inc.) for $20 \mathrm{~min}$ at room temperature in order to determine the amount of PLGA needed to fully self-assemble with siCAP1. Then, the complexes were electrophoresed and analyzed. Samples of PLGA/siCAP1 (500 ng/lane) were loaded in a $4 \%$ agarose gel. The bands on the gel were observed using the UV imaging system (model no. TA-9403; Tianan United Technology Co.).

Characterization of nanoparticles. The sizes of the prepared particles were measured using a Malvern Zetasizer Nano ZS (Malvern Instruments Ltd.) based on dynamic light scattering. The morphologies of the probes were monitored by transmission electron microscopy on a JEOL-2100F instrument (JEOL) under $200 \mathrm{kV}$ of accelerating voltage.

Cell cytotoxicity assays. A549 and H1299 cells were seeded into 96-well plates at 5,000 cells/well. The cells were incubated in $100 \mu 1$ Roswell Park Memorial Institute (RPMI)-1640 culture medium containing $1 \%$ antibiotics (penicillin-streptomycin; Gibco; Thermo Fisher Scientific, Inc.) and 10\% Foundation ${ }^{\mathrm{TM}}$ fetal bovine serum (FBS; Gemini Bio-Products, Inc.) for $24 \mathrm{~h}$ at $37^{\circ} \mathrm{C}$ in a humidified atmosphere containing $5 \% \mathrm{CO}_{2}$. Then, cells were incubated in RPMI-1640 culture medium containing $600 \mu \mathrm{g}$ PLGA/siCAP1, PLGA/Ctrl, PLGA or left untreated in a triple gas incubator for $4 \mathrm{~h}$ at $37^{\circ} \mathrm{C}$. The supernatant was removed after 24 and $48 \mathrm{~h}$, and cell viability was determined by performing the CCK-8 assay. The released formazan product was detected with an Epoch Microplate Spectrometer (BioTek Instruments, Inc.) at an absorbance wavelength of $490 \mathrm{nM}$.

siRNA transfection. A549 cells were untransfected or transfected with PLGA/siCAP1, PLGA, siCAP1 and PLGA/Ctrl in 6-well plates. Cells were seeded into 6-well culture plates $\left(5 \times 10^{4}\right)$ and the following day, the cells were transfected.In the PLGA/siCAP1 group, cells were transfected with PLGA/siCAP1 concentrations [100:1-800:1 (w/w)] 
with siCAP1 at $50 \mathrm{nM}$. Cells in the PLGA/Ctrl group were transfected with PLGA/Ctrl with Ctrl at $50 \mathrm{nM}$, while those in the siCAP1 group were transfected with $50 \mathrm{nM}$ siCAP1 using Lipofectamine ${ }^{\circledR} 2000$ (Invitrogen; Thermo Fisher Scientific, Inc.) according to the manufacturer's protocol. Cells in the PLGA group were transfected with 50 nM PLGA. After 4-6 h of transfection at $37^{\circ} \mathrm{C}$, the medium was replaced with fresh RPMI-1640 medium and after a 24-h transfection at $37^{\circ} \mathrm{C}$, the cells were washed three times in warm PBS and harvested; total RNA or protein was then extracted. Cells incubated in culture media were used as the untreated group.

Western blot analysis. Transfected cells were washed with PBS, harvested and lysed in radioimmunoprecipitation assay buffer with a protease inhibitor cocktail (Roche Applied Science) to isolate total cell proteins. Then, the concentration of protein samples was determined with a Pierce BCA Protein assay kit (Pierce; Thermo Fisher Scientific, Inc.). Equal amounts of protein (30 $\mu \mathrm{g} /$ lane) were separated by SDS-PAGE on a $10 \%$ gel and then transferred to nitrocellulose membrane (EMD Millipore). Following blocking with 5\% non-fat milk for $1 \mathrm{~h}$ at room temperature, the membranes were incubated overnight at $4^{\circ} \mathrm{C}$ with primary antibodies against GAPDH (polyclonal, rabbit anti-human IgG; cat. no. ab9485) and CAP1 (rabbit anti-human IgG; cat. no. ab155079; both 1:1,000; Abcam). After washing the membrane with $1 \mathrm{X}$ PBS containing $0.05 \%$ Tween-20 three times, it were incubated at room temperature for $1 \mathrm{~h}$ with either horseradish peroxidase-conjugated goat anti-rabbit (cat. no. ZB-2301) or goat anti-mouse (cat. no. ZB-2305; both 1:2,000; OriGene Technologies, Inc.) secondary antibodies. The immunoreactive signals for CAP1 were visualized using the enhanced chemiluminescence system from Amersham (GE Healthcare) and subjected to densitometric analyses using ImageJ software (version 1.51; National Institutes of Health). Relative protein expression levels were normalized to GAPDH.

Reverse transcription-quantitative polymerase chain reaction (RT-qPCR) analysis. CAP1 mRNA expression in transfected A549 cells was determined by RT-qPCR. Total RNA was isolated from freshly cultured cells ground in liquid nitrogen was extracted using TRIzol reagent (Invitrogen; Thermo Fisher Scientific, Inc.) and $1 \mu \mathrm{g}$ of total RNA was reverse transcribed into cDNA using a QuantiNova Reverse Transcription kit (Qiagen, Inc.). cDNA templates were then mixed with SYBR Green PCR Master Mix (Toyobo Life Science) and the corresponding primers; qPCR was subsequently performed on the ABI 7500 Real-time PCR System (Applied Biosystems; Thermo Fisher Scientific, Inc.). The thermocycling conditions were set to $95^{\circ} \mathrm{C}$ for $5 \mathrm{~min}$, followed by 40 cycles of $95^{\circ} \mathrm{C}$ for $10 \mathrm{sec}$ and $60^{\circ} \mathrm{C}$ for $30 \mathrm{sec}$. The subsequent dissociation stage was set to $95^{\circ} \mathrm{C}$ for $15 \mathrm{sec}, 60^{\circ} \mathrm{C}$ for $30 \mathrm{sec}$ and $95^{\circ} \mathrm{C}$ for $15 \mathrm{sec}$. Primers were synthesized by Shanghai GenePharma Co., Ltd. The reference gene, GAPDH, was amplified using the following primers: forward, 5'-ACT CGC TGC TTG CTG GTC-3' and reverse, 5'-ATG GGT GCC AAC AAA TCG-3'. The test gene, CAP1, was amplified using the following primers: forward, 5'-GAT TTG TTG GCA CCC ATC TC-3' and reverse, 3'-GCC TGG ATA CTT TCG CTG AC-5'. Data analysis was conducted using the $2^{-\Delta \Delta \mathrm{Cq}}$ method (31) and all RT-qPCR reactions were performed in triplicate.
NSCLC mouse model. The NSCLC mouse model was established through the seeding of A549 cells $\left(5 \times 10^{6}\right)$ under the skins of the right limbs of 20 five-week-old male nude mice (16-20 g) as previously described (21). All mice were kept under specific pathogen-free conditions, at room temperature $\left(20-26^{\circ} \mathrm{C}\right)$ with a humidity level of $50-60 \%$ and a 12-h light/dark cycle. Mice received $5 \mathrm{~g}$ food and $100 \mathrm{ml}$ water per $100 \mathrm{~g}$ body weight per day. Mice were anesthetized with isoflurane mixed with $\mathrm{O}_{2}$. Induction of anesthesia required 2\% isoflurane using Rodent Anesthesia Machine (Shanghai Yuyan Instruments Co., Ltd.). The skin of the mice were disinfected with $70 \%$ alcohol. A549 cells $\left(5 \times 10^{6}\right)$ were suspended in $30 \mu \mathrm{l}$ RPMI-1640 medium. A 29-gauge needle connected to a $0.5-\mathrm{ml}$ syringe containing the A549 cells was directly injected under the skin to a depth $\sim 3 \mathrm{~mm}$. Following confirming their stabilization, the mice were returned to their cages. After 14 days, tumors under the skin were confirmed and the growth was measured every 3 days.

To determine whether PLGA could deliver a sufficient amount of siRNAs to the developing tumors, mice were administered with PLGA/siCAP1 [600:1 (w/w); $1 \mu \mathrm{g}$ siCAP1] via the tail vein. The mice injected with fluorescent siCAP1s alone or PBS were used as controls. A total of $4 \mathrm{~h}$ after the injections, ex vivo fluorescent intensities were measured to evaluate the delivery performances, then mice were sacrificed and dissected. The tumor tissues were fixed, cut into slices and stained. Tumor sections were fixed with $10 \%$ formalin at room temperature for $24 \mathrm{~h}$, dehydrated with different concentrations of ethanol (70,85, 95 and 100\%) and xylene, embedded in paraffin and sliced into $4-\mu \mathrm{m}$-thick sections. Following deparaffinization in xylene, and rehydration in 100, 95, 85 and $70 \%$ ethanol and distilled water, these sections were stained with hematoxylin for $5 \mathrm{~min}$ at room temperature and eosin for $2 \mathrm{~min}$ at room temperature. They were then dehydrated with 70, 85, 95 and $100 \%$ ethanol and xylene, and covered with a coverslip and mounting medium (Sangon Biotech Co., Ltd.). Then they were observed under an inverted light microscope (Nikon Eclipse TS 100; Nikon Corporation) at a magnification of x200. FITC-labeled PLGA/siCAP1 was kept in refrigerator of $4^{\circ} \mathrm{C}$ and in the dark. All experimental protocols were evaluated and approved by the Institutional Review Board for Animal Research at Shanghai Tenth People's Hospital (Shanghai, China).

Transwell assay. For the cell migration assays, chambers with the upper and lower wells were used for cell incubation. Transfected A549 cells $\left(3 \times 10^{4}\right)$ in serum-free RPMI-1640 medium were seeded in the upper chambers, which were equipped with $8-\mu \mathrm{m}$ Transwell membranes (Corning Incorporated, Corning, NY, USA), and $500 \mu \mathrm{l}$ RPMI-1640 medium supplemented with $1 \%$ antibiotics and $10 \%$ FBS was added to the lower chambers. After $24 \mathrm{~h}$ of incubation at a constant temperature of $37^{\circ} \mathrm{C}$, cells were removed from the upper surface using a cotton tip and the chambers were washed thrice with $1 \mathrm{X}$ PBS. Next, the cells that migrated to lower surface of the chamber were fixed with $95 \%$ ethanol for $10 \mathrm{~min}$ at room temperature, stained with $0.1 \%$ crystal violet solution for $10 \mathrm{~min}$ at room temperature, washed thrice in PBS and subsequently air-dried. Then, a light microscope was used to photograph and count these cells in five random fields per 
well (magnification, x100). Cells were counted using Image J software (version 1.51).

Wound healing assay. Cell migration was evaluated by conducting Transwell assays and wound healing assays. A549 cells were cultured in 6-well plates until reaching 95\% confluence. Cells were scratched using a sterile 10- $\mu 1$ pipette tip. Then, wound healing was observed and photographed after 0 and $24 \mathrm{~h}$ using a fluorescent microscope at a magnification of $x 200$. The gap width was quantified with ImageJ software (version 1.51).

Tumor metastasis model. A total of 20 nude mice were randomly assigned into the following four equal groups: Untreated (the blank control group, mice injected with A549 cells), PLGA/Ctrl [the negative control group, mice injected with PLGA/Ctrl-treated A549 cells], PLGA (the control group, mice injected with PLGA-treated A549 cells) and PLGA/siCAP1 (the gene silencing group, mice injected with PLGA/siCAP1-treated A549 cells). Each mouse was intraperitoneally injected with $5 \times 10^{6}$ cells and observed daily. All mice were sacrificed after 14 days and the tumor weights were measured. The nude mice were sacrificed and their abdominal cavities were opened to observe the growth of tumors. Intraperitoneal tumor formation in the internal organs, including the peritoneum, heart, liver, spleen, lung, kidney, intestine, uterus, ovary, diaphragm and omentum, were evaluated in terms of location, the number of tumors, and size of the tumors in the nude mice. The number of tumors with a diameter of $>5 \mathrm{~mm}$ was defined as the tumor number. The number of internal organs with tumor formation was defined as the tumor position. The tumor formation rate was calculated as follows: Tumor formation rate $=$ (number of nude mice with a tumor diameter $>5 \mathrm{~mm} /$ total number of nude mice in the experimental group) x $100 \%$.

Hematoxylin and eosin (H\&E) and immunohistochemical (IHC) assay. Tumor sections were fixed with $10 \%$ formalin at room temperature for $24 \mathrm{~h}$ and dehydrated with 70, 85, 95 and 100\% ethanol and xylene. Then they were embedded in paraffin and sliced into $4-\mu \mathrm{m}$-thick sections. Following deparaffinization in xylene and rehydration 100, 95, 85 and $70 \%$ ethanol and distilled water. These sections were stained with hematoxylin for $5 \mathrm{~min}$ at room temperature and eosin for $2 \mathrm{~min}$ at room temperature, dehydrated with 70, 85, 95 and $100 \%$ ethanol and xylene, and covered with a coverslip and mounting medium (Sangon Biotech Co., Ltd.). The H\&E sections were observed under an inverted light microscope (Nikon Eclipse TS 100; Nikon Corporation) at a magnification of x200 to identify any cancer nests.

For IHC, tissues were fixed in $10 \%$ formalin at room temperature for $24 \mathrm{~h}$ and dehydrated with 70, 85, 95 and $100 \%$ ethanol and xylene. Then they were embedded in paraffin and sliced into $4-\mu \mathrm{m}$-thick sections. Deparaffinization was performed in xylene and rehydration in 100, 95, 85 and $70 \%$ ethanol and distilled water. Then, the sections were soaked in $0.3 \% \mathrm{H}_{2} \mathrm{O}_{2}$ to block the activities of endogenous peroxidases. After washing the sections with $10 \mathrm{mmol} / \mathrm{l}$ citrate buffer ( $\mathrm{pH} 6.0$ ), they were autoclaved for $20 \mathrm{~min}$ at $121^{\circ} \mathrm{C}$ for antigen retrieval. Then, the sections were washed with PBS (pH 7.2) and subsequently incubated with $10 \%$ goat serum (Sangon Biotech Co., Ltd.) at room temperature for $1 \mathrm{~h}$ to prevent the occurrence of non-specific reactions. The sections were incubated overnight with anti-CAP1 antibodies $(1: 500)$ at a constant temperature of $4^{\circ} \mathrm{C}$. The monoclonal mouse anti-CAP1 antibodies were constructed and provided by Professor Jeffrey Field, Department of Systems Pharmacology and Translational Therapeutics, Perelman School of Medicine, University of Pennsylvania School of Medicine (Philadelphia, PA, USA). All sections were processed using the peroxidase-anti-peroxidase method (Dako; Agilent Technologies $\mathrm{GmbH}$ ) (32). The sections were washed with PBS, then incubated in 3,3'-diaminobenzidine tetrahydrochloride hydrate in $0.05 \mathrm{~mol} / 1$ Tris buffer ( $\mathrm{pH}$ 7.6) containing $0.03 \% \mathrm{H}_{2} \mathrm{O}_{2}$ to observe the reaction of peroxidases. Next, the sections were washed with sterile water, counterstained with hematoxylin, dehydrated and added to a cover slip. The counterstained sections were then observed under an inverted light microscope (Nikon Eclipse TS 100) at a magnification of $\times 200$. The sections were observed in $\geq 10$ random high-power fields and $\geq 400$ cells in each field were counted.

Statistical analysis. Each experiment was conducted at least in triplicate. All experimental data are presented as mean \pm standard deviation from at least three independent experiments. One-way analysis of variance with Dunnett's post hoc test was used to analyse the differences among the groups. Statistical analysis was carried out using the SPSS 23.0 software package (IBM Corp.). $\mathrm{P} \leq 0.05$ indicated that the difference between groups was statistically significant.

\section{Results and Discussion}

PLGA/siCAP1 NPs are larger than PLGA NPs and reduce CAP1 mRNA expression. The morphology and structure of PLGA/siCAP1 emulsion were characterized. The average hydrodynamic size of the PLGA emulsion in deionized water was $200 \mathrm{~nm}$ (Fig. 1A and B). The average hydrodynamic size of the PLGA/siCAP1 emulsion in deionized water was $~ 600$ nm (Fig. 1C and D). These results indicate that the addition of siCAP1 increased the emulsion size and that siCAP1 was successfully encapsulated into the PLGA polymers. The expected products were successfully obtained and were further analyzed to investigate their effects on A549 cells.

Agarose gel electrophoresis was conducted to determine the amount of PLGAs needed for full self-assembly with siRNAs mediated by electrostatic interactions. The migration of non-complexed siRNAs was observed at the bottom of the gel as presented in Fig. 2A. Increasing the amount of PLGA complexed to siCAP1 [100:1-800:1 (w/w)] was demonstrated to prevent the migration of the non-complexed siCAP1. These results indicated that increasing the amount of PLGAs to the range of 600:1-800:1 (w/w) promoted the full complex formation of the PLGAs with the siCAP1 (Fig. 2A). As presented in Fig. 2B, an increasing mass of PLGA [100:1-800:1 (w/w); 45.40 \pm 1.664 , $34.53 \pm 3.133,28.23 \pm 1.220,25.60 \pm 1.389,30.07 \pm 1.602$, $26.73 \pm 1.132$, respectively; $\mathrm{P}<0.001]$ combined with a constant mass of siCAP1 (50 nM) was used to transfect A549 cells to investigate whether or not the varying ratios of PLGA influenced 

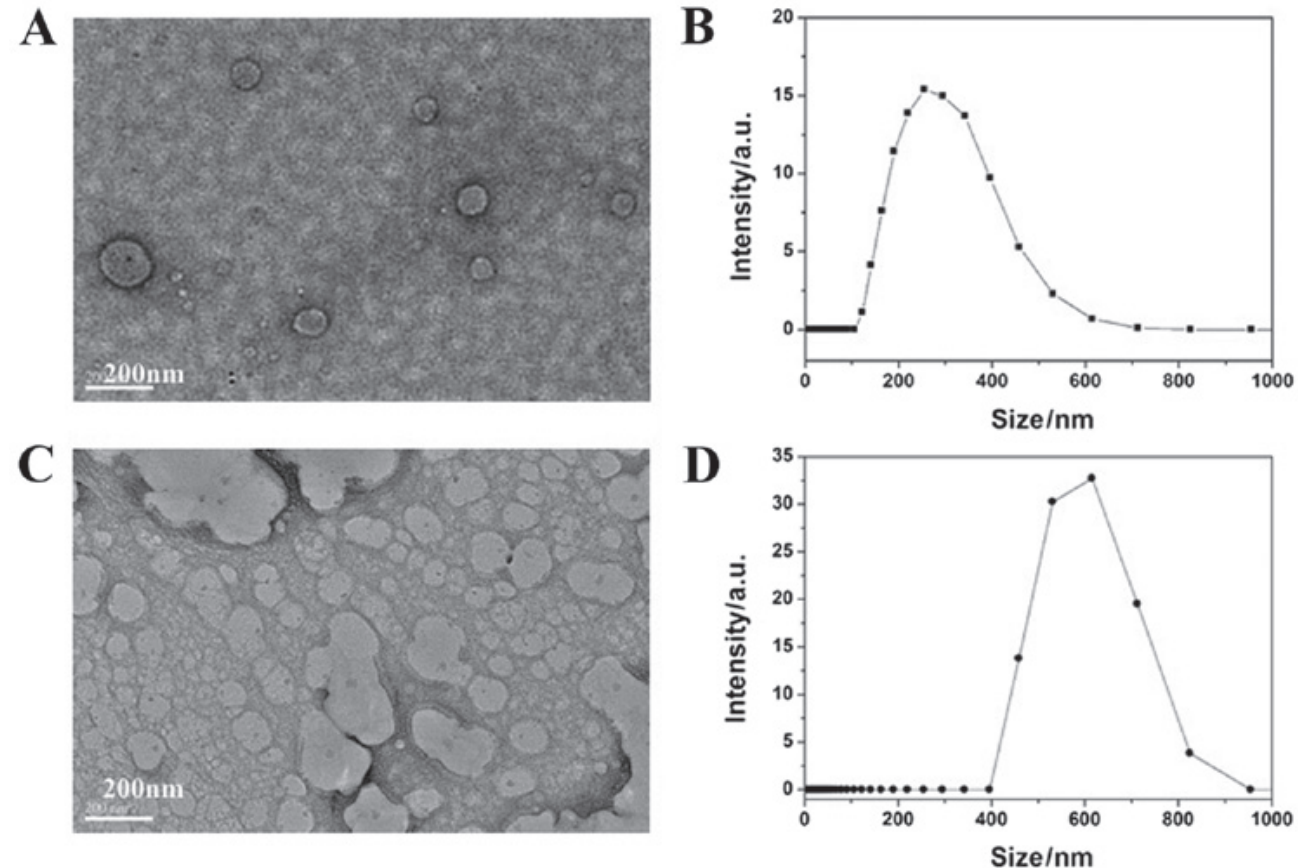

Figure 1. PLGA/siCAP1 NPs are larger than PLGA NPs. (A) TEM images and (B) size distribution graph of PLGA emulsions. (C) TEM images and (D) size distribution graph of PLGA-siCAP1 emulsions. TEM, transmission electron microscopy; PLGA, poly(lactic-co-glycolic acid); CAP1, adenylyl cyclase-associated protein 1; si, small interfering RNA; a.u., arbitrary units; NP, nanoparticle.

A

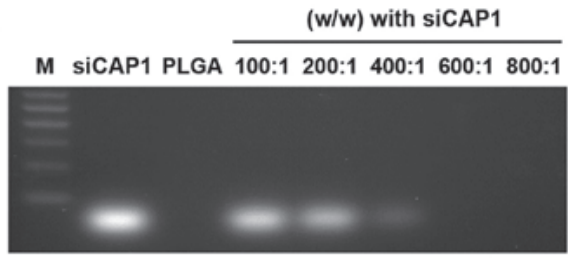

B

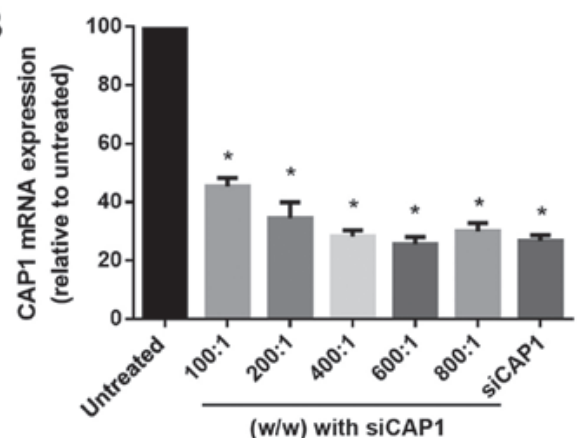

Figure 2. PLGA/siCAP1 NPs reduce CAP1 mRNA expression. (A) Representative agarose gel presenting free unmodified siCAP1 alone (500 ng), PLGA alone (500 ng) and increasing amounts of PLGA complexed to the siCAP1. (B) CAP1 expression in A549 cells after $24 \mathrm{~h}$ of transfection with increasing amounts of PLGA complexed to the siCAP1 (50 nM). Cells incubated in culture media were used as controls. Data are presented as mean \pm standard deviation of three independent experiments. ${ }^{*} \mathrm{P}<0.01$ vs Untreated. M, molecular weight marker; CAP1, adenylyl cyclase-associated protein 1; PLGA, poly(lactic-polyglycolic acid); si, small interfering RNA.

gene silencing. Cells in the control group were treated with culture medium, while other A549 cells were treated with siCAP1 complexed to PLGA or Lipofectamine 2000 (L2K). PLGA/siCAP1 in each selected ratio [100:1-800:1 (w/w)] was demonstrated to effectively silence CAP1. PLGA/siCAP1 in each selected ratio [100:1-800:1 (w/w)] and was demonstrated

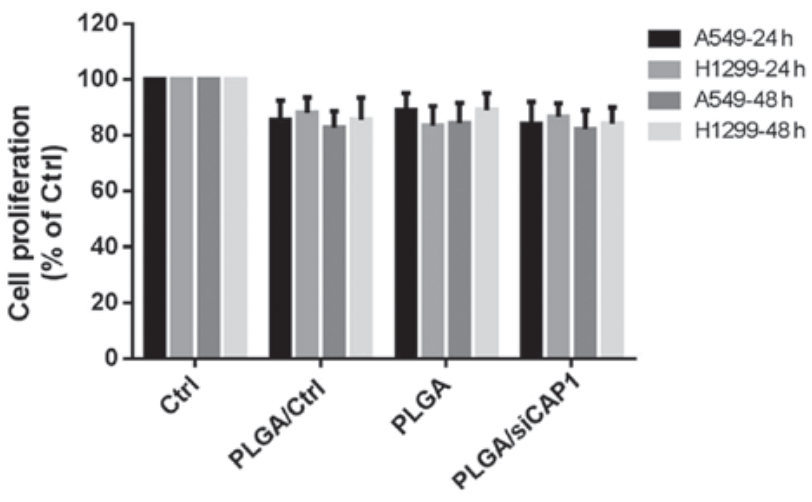

Figure 3. PLGA/siCAP1 nanoparticles have little effect on cell cytotoxicity. The proliferation of A549 and H1299 cells following PLGA and PLGA/siCAP1 nanoparticle treatment. Data are presented as mean \pm standard deviation of three independent experiments. Ctrl, control siRNA; PLGA, poly(lactic-co-glycolic acid); si, small interfering RNA; CAP1, adenylyl cyclase-associated protein 1 .

to effectively silence CAP1 expression in A549 cells as CAP1 mRNA expression was significantly lower in the PLGA/siCAP1 and siCAP1 complexed to L2K groups compared with the control group (all $\mathrm{P}<0.01$; Fig. $2 \mathrm{~B}$ ). When the ratio was adjusted to the range of $600: 1-800: 1(\mathrm{w} / \mathrm{w})$, the siCAP1 significantly inhibited CAP1 expression by over $70 \%$ when compared with the control group. Based on these findings, PLGA/siCAP1 at the ratio of 600:1 (w/w) was demonstrated to be optimal for siRNA delivery in NSCLC cells.

PLGA/siCAP1 NPs have little effect on cell cytotoxicity. CCK-8 assays were conducted to evaluate the cytotoxicity of PLGA and PLGA/siCAP1 at the ratio of 600:1 w/w). Fig. 3 demonstrates the effects of PLGA NPs on the viability of 

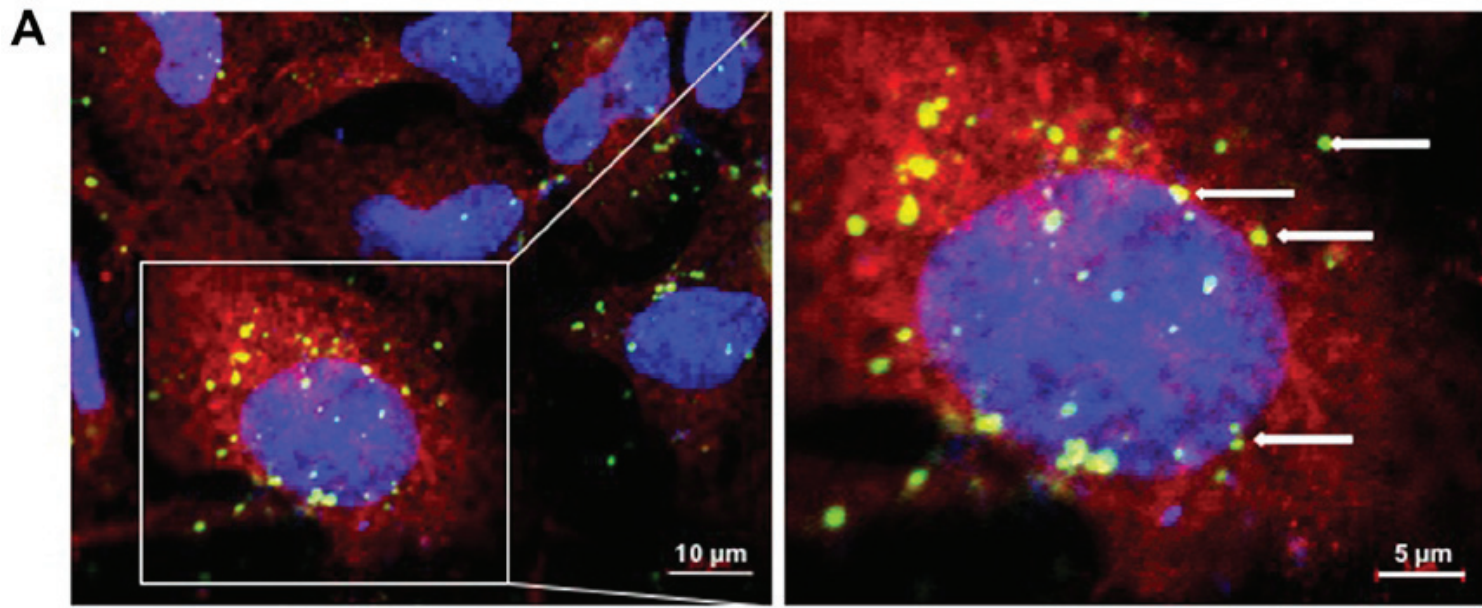

B
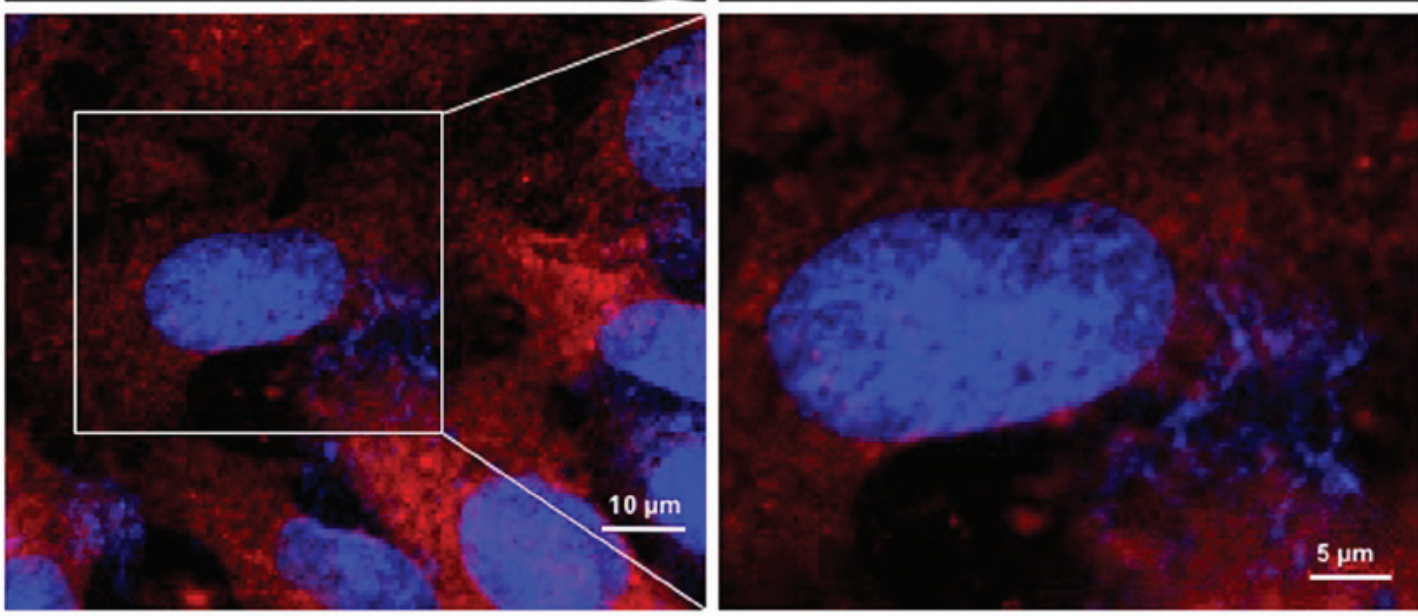

Figure 4. PLGAs deliver siCAP1s to NSCLC cells in vitro. (A) Confocal images of FITC-labeled PLGA/siCAP1 NPs (green), CAP1 (red) and nuclei (blue) in A549 cells. The white arrows reveal the location of PLGA/siCAP1 NPs. (B) Confocal images of FITC-labeled siCAP1s (green) without NPs in A549 cells. NP, nanoparticle; PLGA, poly(lactic-co-glycolic acid); CAP1, adenylyl cyclase-associated protein 1; FITC, fluorescein isothiocyanate; si, small interfering RNA.

A549 and H1299 after $24 \mathrm{~h}$ and $48 \mathrm{~h}$ of treatment. A small, non-significant decrease in cell viability was observed following the addition of the PLGA/siCAP1 emulsion. Approximately $85 \%$ of all $\mathrm{H} 1299$ and A459 cells remained viable after $24 \mathrm{~h}$ and $48 \mathrm{~h}$ of incubation, indicating that the PLGA/siCAP1 emulsion exhibits good biocompatibility and minimal influence on cell viability. Additionally, A549 and H1299 cells treated with PLGA and PLGA/Ctrl exhibited slightly reduced cell viability, which was attributed the mild cytotoxic effects of PLGA.

PLGAs deliver siCAP1s to NSCLC cells in vitro. Previous studies demonstrated the potential use of siCAP1 as effective delivery vehicles for the therapeutic treatment of NSCLC (15-17). Furthermore, PLGA was explored as a delivery system for delivering siRNAs to mice and silencing the expression of key target genes (33-35). Inspired by these previous findings, A549 cells were transfected with PLGA NPs carrying FITC-labeled siCAP1 with the goal of determining the cellular distribution and uptake of PLGA/siCAP1. A549 cells transfected with FITC-labeled siCAP1 were used as the control. After $24 \mathrm{~h}$ of transfection, cellular intake of the siCAP1 was examined by confocal microscopy. The results revealed that siCAP1 is carried by PLGA were efficiently taken up by A549 cells (Fig. 4A). By contrast, siCAP1s alone did not effectively enter the cells (Fig. 4B). Taken together, these results reveal that PLGA NPs can deliver of siCAP1s to NSCLC cells.

PLGA/siCAP1 silences CAP1 expression and reduces the motility of NSCLC cells. Yamazaki et al (36) demonstrated that CAP1 overexpression significantly influenced the aggressiveness of pancreatic cancer. In addition, other studies confirmed that siCAP1s could silence CAP1 expression and reduce the motility of NSCLC cells $(15,16,37)$. Given that PLGA/siCAP1 could deliver siCAP1s to NSCLC cells, the authors of the current study attempted to determine whether PLGA/siCAP1 could silence CAP1 expression and reduce the motility of NSCLC cells; the experiments were conducted using A549 cells treated with PLGAs and PLGA/siCAP1 NPs as the controls. The results indicated that $70 \%$ of CAP1 gene expression levels were inhibited by PLGA/siCAP1 after 48 h of transfection compared with the control groups (PLGA and PLGA/Ctrl; F=114.0; all $\mathrm{P}<0.01$; Fig. 5A). After $72 \mathrm{~h}$ of transfection, CAP1 protein expression was significantly downregulated by $\sim 80 \%$ compared with the control groups $(\mathrm{F}=244.7$; all $\mathrm{P}<0.01$; Fig. 5B). Furthermore, immunofluorescence assays indicated that treatment of A549 cells with PLGA/siCAP1 induced a notable 

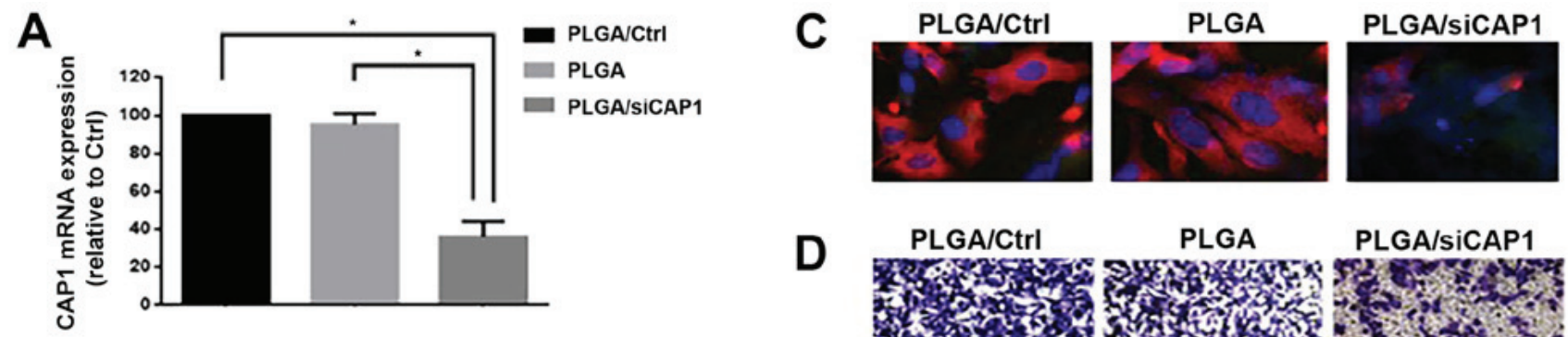

D
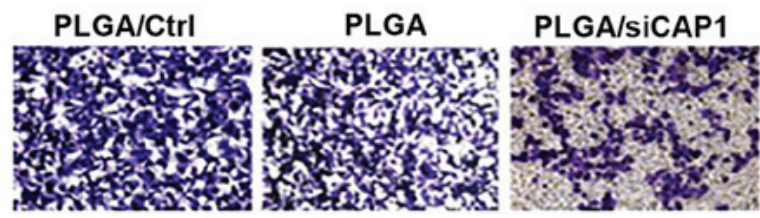

B
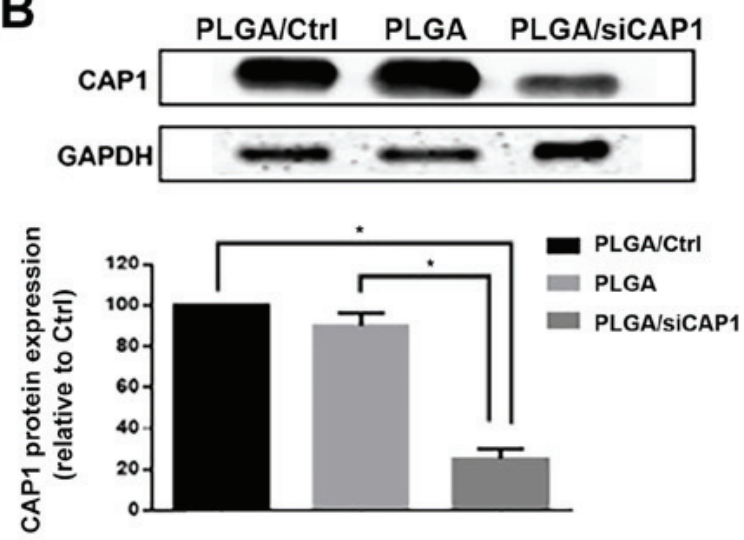
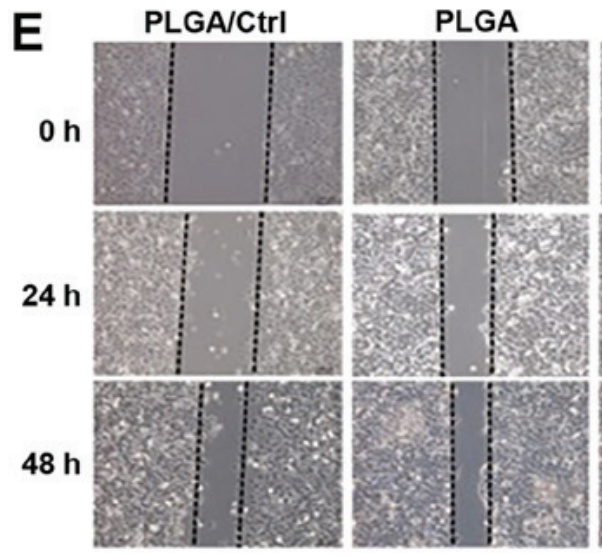

Figure 5. PLGA/siCAP1 silences CAP1 expression and reduces the motility of NSCLC cells in vitro. (A) CAP1 mRNA expression levels cells after 48 h of transfection. (B) Representative agarose gel of a western blot analysis and a densitometry graph of CAP1 protein expression levels $72 \mathrm{~h}$ after transfection. (C) Immunofluorescence images of CAP1 (red) and nuclei (blue). Cell migration (D) 48 h after transfection using Transwell assays and (E) using wound healing assays. Cells treated with PLGA/Ctrl and PLGA alone served as the controls. Data are presented as mean \pm standard deviation of three independent experiments. ${ }^{*} \mathrm{P}<0.01$. CAP1, adenylyl cyclase-associated protein 1; Ctrl, control siRNA; PLGA, poly(lactic-polyglycolic acid); si, small interfering RNA.

decrease in CAP1 expression (Fig. 5C). In addition, the results of Transwell migration assays demonstrated that treatment with PLGA/siCAP1 induced a marked decrease in A549 cell motility (Fig. 5D). The results of the wound healing assays revealed that cell migration was significantly reduced compared with the control groups ( $\mathrm{F}=63.1$; all $\mathrm{P}<0.01$; Figs. 5E and $\mathrm{S} 1 \mathrm{~A})$. Additionally, the results of Transwell assays indicated that cell migration was significantly reduced compared with the control groups ( $\mathrm{F}=288.3$; all $\mathrm{P}<0.01$; Fig. S1B). These results are consistent with the hypothesis of the current study that siCAP1 was capable of suppressing CAP1 expression in A549 cells, which in turn reduced their cell migration capabilities. Taken together, silencing CAP1 expression by PLGA/siCAP1 exerts an anti-metastatic effect on NSCLC cells.

PLGAs deliver siRNAs to NSCLC tumors in vivo. The nude mouse model was established to evaluate the potential therapeutic use of PLGA/siCAP1 in vivo. After 7 days of inoculation with tumor cells, tumors could be detected and measured under the skin. PLGA/siCAP1 group can reduce tumor size as compared to PLGA and untreated group (Figs. 6A, S2 and S3). To determine whether PLGA could deliver a sufficient number of siRNAs to the developing tumors, systemic administration was conducted on the experimental mice with FITC-labeled PLGA/siCAP1 NPs and controls. The collected tumors were observed by confocal microscopy, which verified that the fluorescent siCAP1 was present in the PLGA/siCAP1-treated tumor tissues (Fig. 6B).
No fluorescence in the mice that were injected only with PLGA or PBS was observed, while mice injected with fluorescent siCAP1 demonstrated weak fluorescence signals, which indicated that the siCAP1 was eliminated from the body (Fig. 6C). However, mice injected with PLGA/siCAP1 demonstrated strong fluorescence signals. Furthermore, following treatment with PLGA/siCAP1, CAP1 expression levels in the tumor tissues were markedly reduced (Fig. 6D). Collectively, the findings demonstrated that PLGAs were capable of delivering siCAP1s to NCSLC tissues grown in vivo similar to human clinical settings and successfully downregulated CAP1 expression.

PLGA/siCAP1 reduces NSCLC metastasis in vivo. Given the high expression of CAP1 in tumors grown from implanted human NSCLC cells in nude mice, the potential therapeutic effects of PLGA/siCAP1 on metastatic tumors in vivo was evaluated. The authors of the current study assessed the migration and metastatic abilities of NSCLC tumors by observing tumor growth and inhibition. The four groups of mice experienced intraperitoneal tumor formation in the internal organs, including the peritoneum, heart, liver, spleen, lung, kidney, intestine, uterus, ovary, diaphragm and omentum (Figs. 7 and S4).

The nude mice were sacrificed at 14 days the inoculation with the different vector and the tumors were dissected. The tumor formation rate of PLGA/siCAP1 group was determined to be $60 \%(3 / 5)$ compared with $100 \%(5 / 5), 100 \%(5 / 5)$ and 
Table I. Effects of PLGA/siCAP1 on the growth of NSCLC tumors in nude mice.

\begin{tabular}{llccc}
\hline Group & $N$ & Tumor formation rate & Tumor number & Tumor position \\
\hline Untreated & 5 & $5(100)$ & $7.4 \pm 1.8$ & $4.8 \pm 0.8$ \\
PLGA/Ctrl & 5 & $5(100)$ & $7.8 \pm 1.9$ & $4.8 \pm 0.8$ \\
PLGA & 5 & $4(80)$ & $7.2 \pm 4.2$ & $4.6 \pm 2.6$ \\
PLGA/siCAP1 & 5 & $3(60)$ & $2.3 \pm 3.0^{\mathrm{a}, \mathrm{b}, \mathrm{c}}$ & $1.0 \pm 1.4^{\mathrm{a}, \mathrm{b}, \mathrm{c}}$ \\
\hline
\end{tabular}

Data are presented as $\mathrm{n}(\%)$ or mean \pm standard deviation. ${ }^{\mathrm{a}} \mathrm{P}<0.05$ vs. the untreated group, ${ }^{\mathrm{b}} \mathrm{P}<0.05$ vs. the $\mathrm{PLGA}-\mathrm{Ctrl}$ group, ${ }^{\mathrm{c}} \mathrm{P}<0.05$ vs. the PLGA group. Ctrl, control; PLGA, poly(lactic-co-glycolic acid); Ctrl, control siRNA; CAP1, adenylyl cyclase-associatedprotein 1; NSCLC, non-small cell lung cancer.
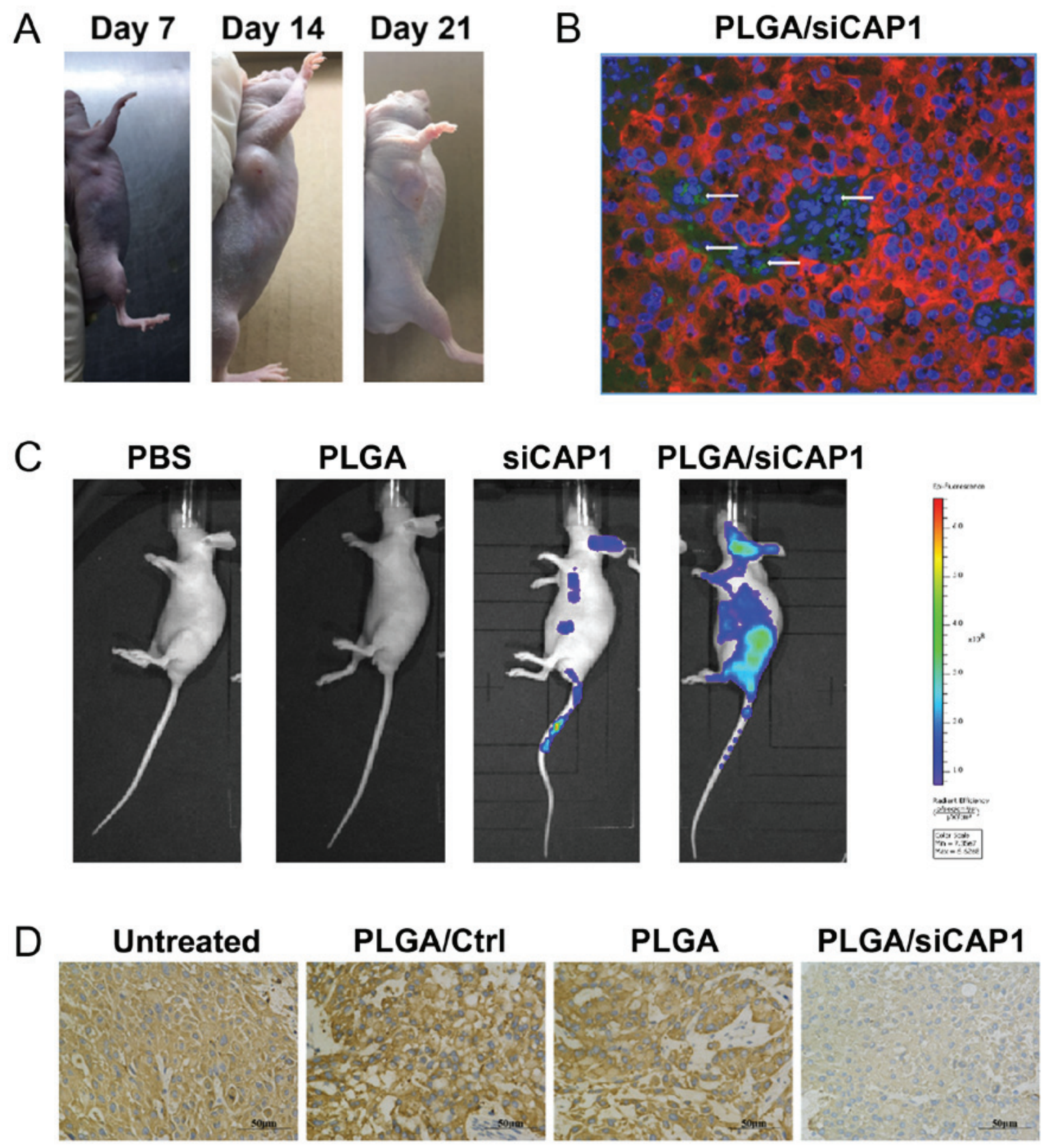

Figure 6. PLGAs deliver siRNAs to NSCLC tumors in vivo. Mice were injected with PBS, PLGA alone and FITC-labeled siCAP1, which served as the controls, and with FITC-labeled PLGA/siCAP1. (A) Images of mice injected with A549 cells. (B) A immunofluorescence image of FITC-labeled PLGA/siCAP1 NPs (green), CAP1 (red) and nuclei (blue) in NSCLC tumor tissues. The white arrows reveal the location of PLGA/siCAP1 NPs. (C) Ex vivo fluorescence images of mice after $4 \mathrm{~h}$ of treatment. (D) Immunohistochemical images of CAP1 expression in tumors in mice. NP, nanoparticle; PLGA, poly(lactic-co-glycolic acid); si, small interfering RNA; CAP1, adenylyl cyclase-associated protein 1; Ctrl, control siRNA; NSCLC, non-small cell lung cancer; FITC, fluorescein isothiocyanate; si, small interfering RNA. 
A

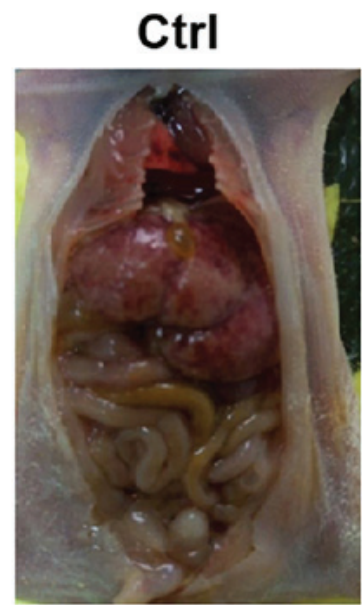

PLGA/Ctrl

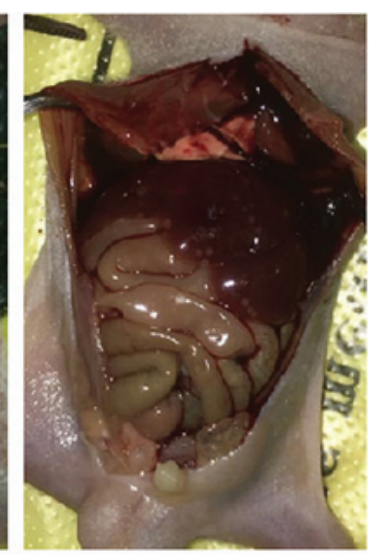

B

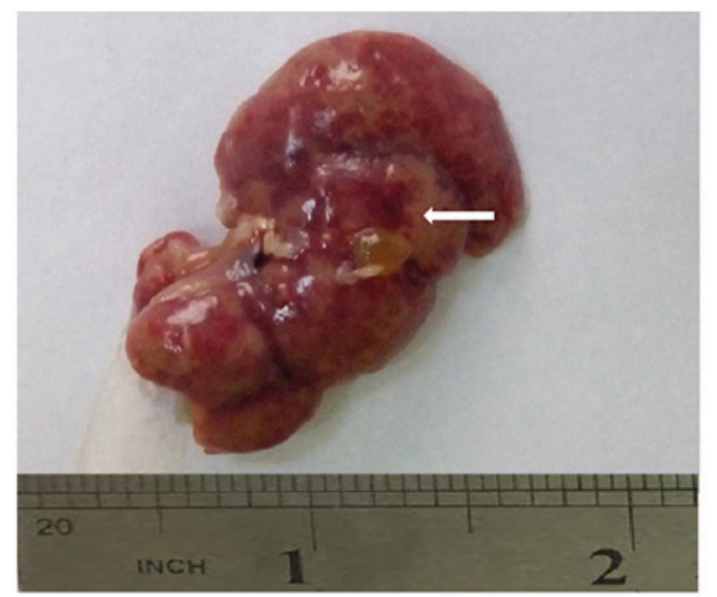

PLGA

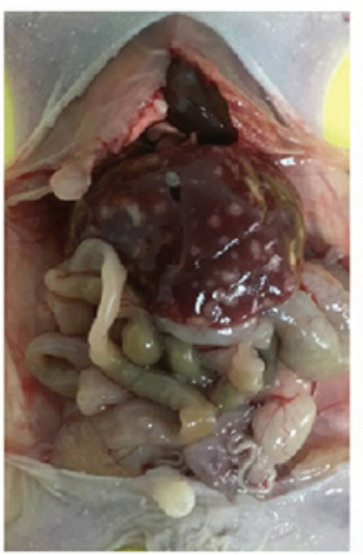

\section{PLGA/siCAP1}

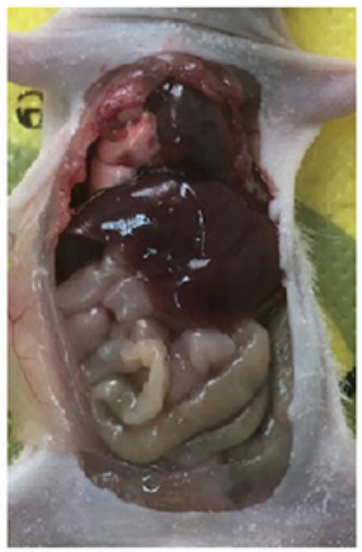

C

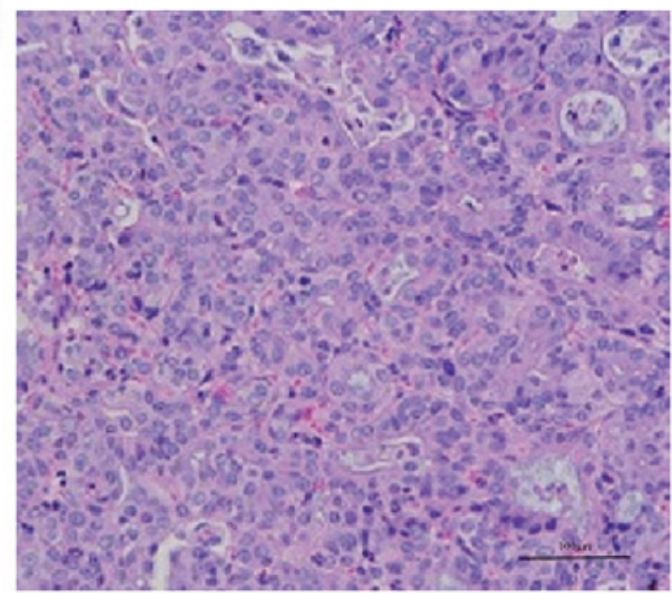

Figure 7. PLGA/siCAP1 reduces non-small cell lung cancer tumor metastasis in vivo. (A) Organs with tumor metastasis. (B) Liver with tumor metastasis. (C) A hematoxlyin and eosin-stained liver tissue section showing tumor metastasis. The white arrows reveal the location of metastasis tumor in the liver. PLGA, poly(lactic-co-glycolic acid); si, small interfering; Ctrl, control siRNA; CAP1, adenylyl cyclase-associated protein 1.

$80 \%(4 / 5)$ in the untreated, PLGA/Ctrl and PLGA groups, respectively (Table I). Although no significant differences in the overall tumor formation rate among the four groups was observed, the sizes of the tumors and number of tumors in the PLGA/siCAP1 group were considerably smaller compared with those in the three other groups. Significant differences in the location of the tumors in mice in the PLGA/siCAP1 group were observed; these tumors were primarily located in the greater omentum and mesentery, whereas the tumors in the mice in the untreated, PLGA/Ctrl and PLGA groups were distributed throughout the omentum, mesentery, liver, renicapsule and heart $(\mathrm{P}<0.05$; Fig. 7 and Table I). Furthermore, no significant differences in the growth of transplanted tumors in mice in the untreated, PLGA/Ctrl, and PLGA groups were observed.

In the present study, PLGA/siCAP1 NPs were prepared and used as macromolecule carriers for the targeted delivery of siRNAs against CAP1 to regulate CAP1 expression in vivo and in vitro. The results demonstrated that silencing CAP1 expression using PLGA/siCAP1 NPs could markedly inhibit the migration of A549 cells in vivo and in vitro. To the best of our knowledge, the current study is the first to demonstrate the successful delivery of siCAP1s to NSCLC cells using PLGAs, which reduced the migration of tumor cells in a metastatic
NSCLC mouse model that can mimic the clinical tumor microenvironment.

The findings of the current study provided a basis for the development of a novel strategy of drug delivery by PLGA-mediated delivery of siCAP1s to NSCLC cells in vivo and in vitro. In addition, PLGA/siCAP1s effectively silenced CAP1 expression in NSCLC cells, and reduced cell motility and metastatic potential. The current study demonstrated the potential use of PLGA/siCAP1 for the therapeutic treatment of metastatic NSCLC. Additionally, the proposed method described in the current study could be applied to other types of cancers characterized by CAP1 expression.

\section{Acknowledgements}

Not applicable.

\section{Funding}

This work was supported by the National Natural Science Foundation of China (grant nos. 81472180 and 81802262) and Shanghai Tenth Hospital's improvement plan for NSFC (grant nos. 04.03.17.032 and 04.01.18.048). 


\section{Availability of data and materials}

All data generated or analyzed during this study are included in this published article.

\section{Authors' contributions}

YL and SX collected and analyzed the data. YL, MT and JZ performed the experiments and wrote the manuscript. SX, JZ, PW, JW and XS designed the study and wrote of the manuscript. $\mathrm{CW}$ and DH conceived the study, directed the project and contributed to writing of the manuscript. All authors edited and approved the final version of the manuscript.

\section{Ethics approval and consent to participate}

Animal experiments were approved by the Institutional Review Board for Animal Research at Shanghai Tenth People's Hospital (Shanghai, China).

\section{Patient consent for publication}

Not applicable.

\section{Conflicts of interest}

The authors declare that they have no conflict of interest.

\section{References}

1. Torre LA, Bray F, Siegel RL, Ferlay J, Lortet-Tieulent J and Jemal A: Global cancer statistics, 2012. CA Cancer J Clin 65: 87-108, 2015.

2. Ramalingam SS, Owonikoko TK and Khuri FR: Lung cancer: New biological insights and recent therapeutic advances. CA Cancer J Clin 61: 91-112, 2011.

3. Aisner DL and Marshall CB: Molecular pathology of non-small cell lung cancer: A practical guide. Am J Clin Pathol 138 332-346, 2012.

4. Lozano R, Naghavi M, Foreman K, Lim S, Shibuya K, Aboyans V, Abraham J, Adair T, Aggarwal R, Ahn SY, et al: Global and regional mortality from 235 causes of death for 20 age groups in 1990 and 2010: A systematic analysis for the Global Burden of Disease Study 2010. Lancet 380: 2095-2128, 2012.

5. Fedor-Chaiken M, Deschenes RJ and Broach JR: SRV2, a gene required for RAS activation of adenylate cyclase in yeast. Cell 61: 329-340, 1990.

6. Field J, Vojtek A, Ballester R, Bolger G, Colicelli J, FergusonK, Gerst J, Kataoka T, Michaeli T, Powers S, et al: Cloning and characterization of CAP, the $S$. cerevisiae gene encoding the $70 \mathrm{kd}$ adenylyl cyclase-associated protein. Cell 61: 319-327, 1990.

7. Matviw H, Yu G and Young D: Identification of a human cDNA encoding a protein that is structurally and functionally related to the yeast adenylyl cyclase-associated CAP proteins. Mol Cell Biol 12: 5033-5040, 1992.

8. Freeman NL, Chen Z, Horenstein J, Weber A and Field J: An actin monomer binding activity localizes to the carboxyl-terminal half of the Saccharomyces cerevisiae cyclase-associated protein. J Biol Chem 270: 5680-5685, 1995.

9. Moriyama K and Yahara I: Human CAP1 is a key factor in the recycling of cofilin and actin for rapid actin turnover. J Cell Sci 115: 1591-1601, 2002

10. Hubberstey AV and Mottillo EP: Cyclase-associated proteins: CAPacity for linking signal transduction and actin polymerization. FASEB J 16: 487-499, 2002.

11. Loisel TP, Boujemaa R, Pantaloni D and Carlier MF: Reconstitution of actin-based motility of Listeria and Shigella using pure proteins. Nature 401: 613-616, 1999.
12. Yu XF, Ni QC, Chen JP, Xu JF, Jiang Y, Yang SY, Ma J, Gu XL, Wang $\mathrm{H}$ and Wang YY: Knocking down the expression of adenylate cyclase-associated protein 1 inhibits the proliferation and migration of breast cancer cells. Exp Mol Pathol 96: 188-194, 2014.

13. Fan YC, Cui CC, Zhu YS, Zhang L, Shi M, Yu JS, Bai J and Zheng JN: Overexpression of CAP1 and its significance in tumor cell proliferation, migration and invasion in glioma. Oncol Rep 36: 1619-1625, 2016.

14. Hua M, Yan S, Deng Y, Xi Q, Liu R, Yang S, Liu J, Tang C, Wang Y and Zhong J: CAP1 is overexpressed in human epithelial ovarian cancer and promotes cell proliferation. Int J Mol Med 35: 941-949, 2015.

15. Xie SS, Tan M, Lin HY, Xu L, Shen CX, Yuan Q, Song XL and Wang $\mathrm{CH}$ : Overexpression of adenylate cyclase-associated protein 1 may predict brain metastasis in non-small cell lung cancer. Oncol Rep 33: 363-371, 2015.

16. Xie S, Shen C, Tan M, Li M, Song X and Wang C: Systematic analysis of gene expression alterations and clinical outcomes of adenylate cyclase-associated protein in cancer. Oncotarget 8: 27216-27239, 2017.

17. Tan M, Song X, Zhang G, Peng A, Li X, Li M, Liu Y and Wang C: Overexpression of adenylate cyclase-associated protein 1 is associated with metastasis of lung cancer. Oncol Rep 30: 1639-1644, 2013.

18. Rana TM: Illuminating the silence: Understanding the structure and function of small RNAs. Nat Rev Mol Cell Biol 8: 23-36, 2007.

19. Coelho T, Adams D, Silva A, Lozeron P, Hawkins PN, Mant T, Perez J, Chiesa J, Warrington S, Tranter E, et al: Safety and efficacy of RNAi therapy for transthyretin amyloidosis. N Engl J Med 369: 819-829, 2013.

20. Davis ME, Zuckerman JE, Choi CH, Seligson D, Tolcher A, Alabi CA, Yen Y, Heidel JD and Ribas A: Evidence of RNAi in humans from systemically administered siRNA via targeted nanoparticles. Nature 464: 1067-1070, 2010.

21. Tabernero J, Shapiro GI, LoRusso PM, Cervantes A, Schwartz GK, Weiss GJ, Paz-Ares L, Cho DC, Infante JR, Alsina M, et al: First-in-humans trial of an RNA interference therapeutic targeting VEGF and KSP in cancer patients with liver involvement. Cancer Discov 3: 406-417, 2013.

22. Qi L, Shao W and Shi D; QiLingfeng: Shao W and Shi D: JAM-2 siRNA intracellular delivery and real-time imaging by proton-sponge coated quantum dots. J Mater Chem B Mater Biol Med 1: 654-660, 2013

23. Su J, Baigude H, McCarroll J and Rana TM: Silencing microRNA by interfering nanoparticles in mice. Nucleic Acids Res 39: e38, 2011.

24. Sezlev Bilecen D, Rodriguez-Cabello JC, Uludag H and Hasirci V: Construction of a PLGA based, targeted siRNA delivery system for treatment of osteoporosis. J Biomater Sci Polym Ed 28: 1859-1873, 2017.

25. Pisani E, Tsapis N, Paris J, Nicolas V, Cattel L and Fattal E: Polymeric nano/microcapsules of liquid perfluorocarbons for ultrasonic imaging: Physical characterization. Langmuir 22: 4397-4402, 2006.

26. Patnaik S, Sharma AK, Garg BS, Gandhi RP and Gupta KC: Photoisomerization of azobenzene moiety in crosslinking. Int $\mathrm{J}$ Pharm 342: 184-193, 2007.

27. Cheng J, Teply BA, Sherifi I, Sung J, Luther G, Gu FX, Levy-Nissenbaum E, Radovic-Moreno AF, Langer R and Farokhzad OC: Formulation of functionalized PLGA-PEG nanoparticles for in vivo targeted drug delivery. Biomaterials 28 : 869-876, 2007.

28. Díaz-López R, Tsapis N, Santin M, Bridal SL, Nicolas V, Jaillard D, Libong D, Chaminade P, Marsaud V, Vauthier C, et al: The performance of PEGylated nanocapsules of perfluorooctyl bromide as an ultrasound contrast agent. Biomaterials 31: 1723-1731, 2010.

29. Boyer C, Teo J, Phillips P, Erlich RB, Sagnella S, Sharbeen G, Dwarte T, Duong HT, Goldstein D, Davis TP, et al: Effective delivery of siRNA into cancer cells and tumors using well-defined biodegradable cationic star polymers. Mol Pharm 10: 2435-2444, 2013.

30. Byrne FL, Yang L, Phillips PA, Hansford LM, Fletcher JI, Ormandy CJ, McCarroll JA and Kavallaris M: RNAi-mediated stathmin suppression reduces lung metastasis in an orthotopic neuroblastoma mouse model. Oncogene 33: 882-890, 2014.

31. Livak KJ and Schmittgen TD: Analysis of relative gene expression data using real-time quantitative PCR and the 2(-Delta Delta C(T)) Method. Methods 25: 402-408, 2001. 
32. van der Weij JP, van der Veen CJ, de Vries E and Cats A: The use of a peroxidase-anti-peroxidase complex for the visualization of monoclonal antibodies on the ultrastructural level. Clin Exp Immunol 54: 819-826, 1983.

33. McCarroll JA, Gan PP, Liu M and Kavallaris M: betaIII-tubulin is a multifunctional protein involved in drug sensitivity and tumorigenesis in non-small cell lung cancer. Cancer Res 70: 4995-5003, 2010.

34. De Rosa G and Salzano G: PLGA microspheres encapsulating siRNA. Methods Mol Biol 1218: 43-51, 2015.

35. Zhao Y, Zheng C, Zhang L, Chen Y, Ye Y and Zhao M: Knockdown of STAT3 expression in SKOV3 cells by biodegradable siRNA-PLGA/CSO conjugate micelles. Colloids Surf B Biointerfaces 127: 155-163, 2015.
36. Yamazaki K, Takamura M, Masugi Y, Mori T, Du W, Hibi T, Hiraoka N, Ohta T, Ohki M, Hirohashi S, et al: Adenylate cyclase-associated protein 1 overexpressed in pancreatic cancers is involved in cancer cell motility. Lab Invest 89: 425-432, 2009.

37. Frede A, Neuhaus B, Klopfleisch R, Walker C, Buer J, Müller W, Epple M and Westendorf AM: Colonic gene silencing using siRNA-loaded calcium phosphate/PLGA nanoparticles ameliorates intestinal inflammation in vivo. J Control Release 222: 86-96, 2016.

c) (1) $(9$ This work is licensed under a Creative Commons EY No No Attribution-NonCommercial-NoDerivatives 4.0 International (CC BY-NC-ND 4.0) License. 